\title{
Comparison of Model-Based and Non-Model-Based Strategies for Nonlinear Control of a Three-Tank System
}

\author{
Martin A. Capcha, William Ipanaqué \\ Laboratory of Automatic Control Systems \\ Piura University \\ Piura, Perú \\ martin.capcha@posgrado.udep.edu.pe, \\ william.ipanaque@udep.pe
}

\author{
Robin De Keyser \\ Department of Electrical Energy, Systems and Automation \\ Ghent University \\ Gent, Belgium \\ Robain.DeKeyser@UGent.be
}

\begin{abstract}
To achieve optimal performance in the regulation of nonlinear systems, more advanced control techniques are needed. In this paper, three of these techniques are applied and compared in a system composed of three tanks in cascade. The first one is the Nonlinear Extended Prediction Self-Adaptive Control (NEPSAC). NEPSAC is a nonlinear predictive controller and therefore, is based on solving an optimization problem using the nonlinear model of the process to find a feedback control law. The second one is a gain scheduling controller using an array of autotuned PID controllers. This will be the non-model-based strategy. Finally, the gain scheduling approach is used again but with an array of linear model predictive controllers. In this case the predictive controller is EPSAC, the linear version of NEPSAC controller. Comparing all these control strategies, this work deepens in the characteristics, performance, advantages and disadvantages of these techniques to deal with nonlinear processes. It is intended to be a reference guide to choose - from a practical point of view - the most appropriate controller when dealing with a soft nonlinear system.
\end{abstract}

Keywords- Nonlinear predictive control, Adaptive control, Output regulation

\section{INTRODUCTION}

Most of the relevant processes in the industry present nonlinearities in their behavior and in many cases, these nonlinearities cannot be avoided. The use of the very wellknown linear control techniques have demonstrated their limitations in dealing with these kind of processes, and the development and applications of nonlinear control techniques are necessary for a good performance in the processes.

One alternative is Nonlinear Model Predictive Control (NMPC), which is a specialized set of control strategies that are part of the more general Model Predictive Control (MPC) family of controllers. NMPC is based in the on-line calculation of the control action in the attempt to optimize a cost function using the mathematical model of the system [1]. However, the special feature of NMPC controllers is that they can handle nonlinear models of the different processes and, in this way, they are not limited to processes with a single point of operation, or processes with low nonlinear behavior; their use is justified when the process is strongly nonlinear or when the system needs to work in different operating zones and regimes [2].

Although NMPC theory had reached a certain degree of maturity in the last years, it is still limited to a certain kind of applications. This restriction is in part because of the lack of a guaranteed real-time solution of the resulting nonlinear optimization problem due to the multiple local minima the problem could have, and also because of the difficulty to create a nonlinear model of the system, together with nonlinear state estimators of the same [3].

In this paper, the NMPC technique applied will be Nonlinear Extended Prediction Self-Adaptive Control (NEPSAC) [4]. NEPSAC is a nonlinear control technique, used in different works as in [5] or [6]. It is characterized by using a nonlinear model of the system instead of a linear approximation around an operating point, and it uses an iterative method for the optimization of the predicted behavior of the system, making its implementation computationally friendly compared to other nonlinear predictive control techniques.

Another popular choice is the use of gain scheduling control, which has long acceptance to deal with nonlinear systems [7]. This method uses an array of linear controllers optimized around specific operating points of the system, and a gain scheduling signal, which will control and generate the control signal based on the individual responses of each linear controller. This way, the gain scheduling control can handle the variations of the dynamics of the system in its entire operation range.

The second method used in this work is thus gain scheduling with an array of autotuned PID controllers (GS-PID). This technique does not need any model of the process because the parameters of each PID controller are tuned automatically based on the response of the system to the application of simple tests.

The third technique, called here GS-EPSAC, will also be a gain scheduling approach but using an array of linear model predictive controllers, specifically the Extended Prediction SelfAdaptive Control (EPSAC), presented in [4] and [8]. With this technique, the facilities to design linear controllers is combined 
with the advantages that model predictive control gives to the controlled system.

The nonlinear process studied in this work consists of three tanks in cascade and the content of this paper is as follows. The description of the equations that govern this process is presented in section II. In section III, EPSAC and NEPSAC controllers' procedure is described. In section IV, the three mentioned nonlinear strategies are presented. In section $\mathrm{V}$ the performances of these control strategies are compared. Finally, section VI concludes this work making some remarks regarding the advantages and disadvantages of the implementation of each approach studied.

\section{PROCESS DESCRIPTION}

The process in this paper consists of three water tanks in cascade, as in Fig. 1. This system has one manipulated input $(u)$, which is a water flow towards the first tank, one not manipulated input (d) as a disturbance on the second tank, and a single controlled output $(y)$ which is the outflow of water of the third tank.

\section{A. Nonlinear process}

Using Torricelli's theorem, it is known that the water level in a tank discharged only by effect of gravity responds to the equation in (1), where $h$ is the water level of the tank, $q_{\text {in }}$ is the flow of water towards the tank, $q_{\text {out }}$ is the outflow of the tank, $A$ is the area of the tank's section, $A_{s}$ is the area of the outlet, $C_{d}$ is the discharge coefficient, and $g$ is the gravity.

$$
\begin{gathered}
A \frac{d(h(t))}{d t}=q_{\text {in }}(t)-q_{\text {out }}(t) \\
q_{\text {out }}(t)=\frac{\sqrt{h(t)}}{R} \\
R=\frac{1}{\sqrt{2 g} C_{d} A_{s}}
\end{gathered}
$$

For simplicity of simulation, and without loss of generality, all the tanks in the process have the same characteristics with their values of $A$ and $R$ equal to 1 . Using these assumptions, the equation in (1) and the Fig. 1, the mathematical model of the system of three tanks is shown in (2). This model does not consider the disturbance on the second tank because it is considered as a non-modeled non-measurable disturbance. Fig. 2 shows the response of the system to step signals at the input of the same amplitude at 5 different initial outputs, showing its

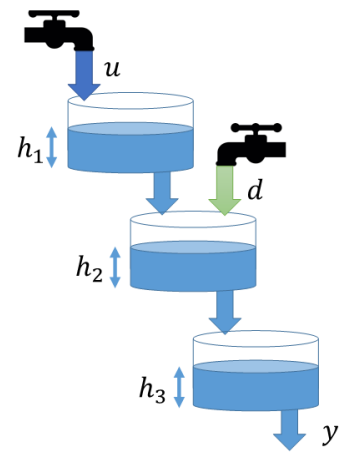

Fig. 1. System of three tanks in cascade with one manipulated input, one controlled output and one disturbance strong nonlinear behavior with the great changes in the settling time, which goes from 240 seconds to 1440 seconds.

$$
\begin{gathered}
\frac{d\left(h_{1}(t)\right)}{d t}=u(t)-\sqrt{h_{1}(t)} \\
\frac{d\left(h_{2}(t)\right)}{d t}=\sqrt{h_{1}(t)}-\sqrt{h_{2}(t)} \\
\frac{d\left(h_{3}(t)\right)}{d t}=\sqrt{h_{2}(t)}-\sqrt{h_{3}(t)} \\
y(t)=\sqrt{h_{3}(t)}
\end{gathered}
$$

For the NEPSAC-NMPC model, a discretized version of (2) has been used:

$$
\begin{gathered}
h_{1}(t)=\left[-\frac{T_{s}}{2}+\sqrt{\left(\frac{T_{s}}{2}\right)^{2}+h_{1}(t-1)+T_{s} u(t-1)}\right]^{2} \\
h_{2}(t)=\left[-\frac{T_{s}}{2}+\sqrt{\left(\frac{T_{s}}{2}\right)^{2}+h_{2}(t-1)+T_{s} \sqrt{h_{1}(t)}}\right]^{2} \\
h_{3}(t)=\left[-\frac{T_{s}}{2}+\sqrt{\left(\frac{T_{s}}{2}\right)^{2}+h_{3}(t-1)+T_{s} \sqrt{h_{2}(t)}}\right]^{2} \\
y(t)=\sqrt{h_{3}(t)}
\end{gathered}
$$

In (3), $t$ is the discrete-time index (sample number) and $T_{s}$ is the sampling time (10 seconds).

\section{B. Process linearization}

Because the third technique (GS-EPSAC) is based on an array of linear model predictive controllers, it is necessary to have linear models which fit the process in specific operating points.

First in (2), it is make the change of variables $h_{1}=H_{1}^{2}$ and $h_{2}=H_{2}^{2}$, converting (2) in (4).

$$
\begin{gathered}
2 H_{1} H_{1}^{\prime}=u-H_{1} \\
2 H_{2} H_{2}^{\prime}=H_{1}-H_{2} \\
2 y y^{\prime}=H_{2}-y
\end{gathered}
$$

In steady state, the variations are null and the signals take a constant value $\left(u=\bar{u}, H_{1}=\bar{H}_{1}, H_{2}=\bar{H}_{2}\right)$ then:

$$
\bar{u}=\bar{H}_{1}=\bar{H}_{2}=\bar{y} \text {. }
$$

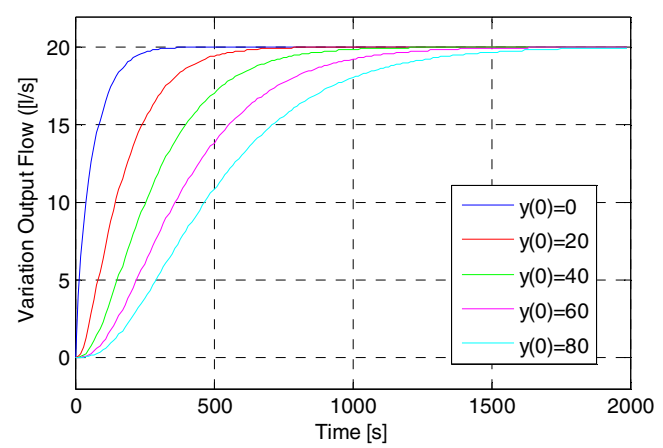

Fig. 2. Response of the system to input steps of the same amplitude at different initial heights 
If every term in (4) is composed of two components, one constant and one variable (e.g. $u=\bar{u}+\Delta u$ ), (4) is converted in (6) using this two components and (5), neglecting the nonlinear terms of the variable components (e.g. $\Delta H_{1} \Delta H_{1}^{\prime}$ ).

$$
\begin{aligned}
2 \bar{H}_{1} \Delta H_{1}^{\prime} & =\Delta u-\Delta H_{1} \\
2 \bar{H}_{2} \Delta H_{2}^{\prime} & =\Delta H_{1}-\Delta H_{2} \\
2 \bar{y} \Delta y^{\prime} & =\Delta H_{2}-\Delta y
\end{aligned}
$$

Finally, the transfer function that relates the variables $\Delta u$ and $\Delta y$, which are the variations around the operating point of the input and output, respectively, is described in (7).

$$
\frac{\Delta y(s)}{\Delta u(s)}=\frac{1}{(2 \bar{y} s+1)^{3}}
$$

This transfer function changes with the outflow and with higher values of this, the system's dynamics is slower, which is in accordance with Fig. 2. This is why the process output signal has been selected as the scheduling signal in the 2 gain scheduling methods (GS-PID and GS-EPSAC).

\section{CONTROL TECHNIQUES APPLIED}

\section{A. Gain scheduled PID controller}

The gain scheduling approach using PID controllers is illustrated in Fig. 3 and it is the non-model-based technique used in this work. The process output is used as the scheduling signal. The controller scheduler will interpolate the outputs of the PID controllers depending on the level of this scheduling signal like the relationship (8) in order to obtain the control signal.

$$
u=\left\{\begin{array}{c}
u_{P I D \sim 80}, \quad 80 \leq y \\
\frac{(80-y) u_{P I D \sim 50}+(y-50) u_{P I D \sim 80}}{30}, 50 \leq y<80 \\
\frac{(50-y) u_{P I D \sim 20}+(y-20) u_{P I D \sim 50}}{30}, 20 \leq y<50 \\
u_{P I D \sim 20}, \quad y<20
\end{array}\right.
$$

Three PID controllers were used and designed to deal with the whole operating range of the process and where sufficient to not lose similarity in the responses of the different tests. The first has been tuned around an output of $20 \mathrm{l} / \mathrm{s}$ with a phase margin of $50^{\circ}$, the second around an output of $50 \mathrm{l} / \mathrm{s}$ with a phase margin of $40^{\circ}$, and the last around an output of $801 / \mathrm{s}$ with a phase margin of $30^{\circ}$. For a quick but reliable design of the PID controllers, a relay-based autotuner described in [9] has been used. This autotuning method creates an estimation of the Nyquist diagram of the process, designing the parameters of the PID controller based on this information and the required phase margin of the closed loop system.

\section{B. NEPSAC controller}

The NEPSAC controller uses the explicit nonlinear model of the process described in (3). For this case, a prediction horizon of $15\left(N_{2}=15\right)$ has been used in order to have a fast response to changes in the setpoint reference and disturbances. Also, to obtain a fast response without too big changes in the manipulated variable, the parameter $\alpha$ of this approach has a value of 0.5 . The control horizon is equal to $1\left(N_{u}=1\right)$.

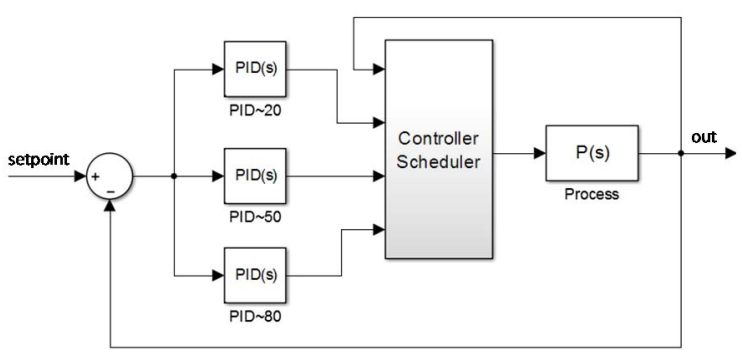

Fig. 3. Gain scheduling with PID controllers

\section{Gain scheduled EPSAC controller}

The gain scheduling with EPSAC controllers is the third control technique used in this work. This technique combines the advantages of predictive control - such as easy dealing with constraints on the system signals - with the advantages of the gain scheduling approach, and with an implementation similar to the first control technique but replacing PID controllers with EPSAC controllers in Fig. 3. Unlike GS-PID, this GS-EPSAC approach needs an explicit set of linear models of the process because of the many predictive controllers used. These linear models are obtained from the linear approximation of the system in (7). Similarly to the gain scheduling technique with PID controllers, three EPSAC controllers are used, with the linear models around the outputs $20 \mathrm{l} / \mathrm{s}, 50 \mathrm{l} / \mathrm{s}$, and $80 \mathrm{l} / \mathrm{s}$. The controller scheduler is the same as for the first method, using the process output as scheduling signal. The linear EPSAC controllers will have the same parameters of the NEPSAC controller for a better comparison and also the same rule of interpolation of the GS-PID controller to calculate the control signal showed in (8).

\section{COMPARISON BETWEEN CONTROL TECHNIQUES}

Comparisons of the 3 approaches are given in Figs. 4-5-6. These tests cover the whole operating range of this nonlinear system. The only restriction in the process is the maximum value of the input flow, which is $200 \mathrm{l} / \mathrm{s}$. The unmeasurable disturbance on the second tank will have a change in its value from $0 \mathrm{l} / \mathrm{s}$ to $-20 \mathrm{l} / \mathrm{s}$ in the middle of each test to observe the performance of the controllers rejecting disturbances.

In the Fig. 4 , the output reference is $20 \mathrm{l} / \mathrm{s}$. In this figure, the settling time of the output using the NEPSAC controller is just
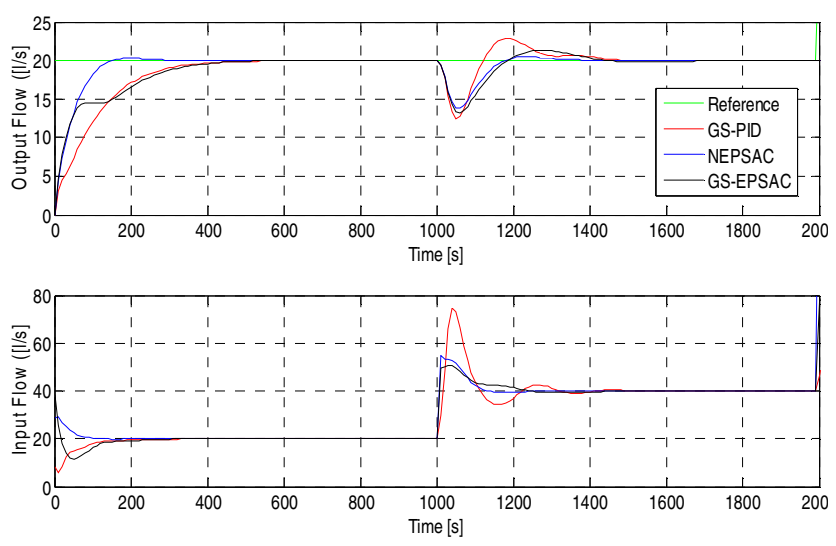

Fig. 4. Comparison of the nonlinear controllers when the reference value is 20 
130 seconds, while for the GS-PID and GS-EPSAC controllers is three times bigger. Similar settling times occur after the disturbance of $-201 / \mathrm{s}$ at time 1000 seconds. Something to highlight here is that although the active PID controller in the GS-PID approach is the one designed around an output of 20 and with the highest phase margin, the settling time is the lower of the three tests. This is because the nonlinearity of the system changes its dynamics notoriously, as can be seen in Fig. 2, where the dynamics near 0 are faster than with an initial output of 20 , and therefore changing also the dynamics in closed loop. In the Fig. 5, the setpoint is $60 \mathrm{l} / \mathrm{s}$ and similar to the previous test, the best performance comes from the NEPSAC controller, with a settling time of 370 seconds, while for the other two, the settling time is around 700 seconds, almost the double. About rejecting the disturbance, the performance of the GS-EPSAC implementation is now more similar to the performance of the NEPSAC controller. Finally, the Fig. 6 presents the performance of the different approaches for a setpoint of 100 1/s. Again, the NEPSAC controller exhibit the best behavior of the three, both to reach the setpoint and to reject the disturbance.

To finish the comparison of the performances, the Table I summarizes the medium square error (MSE) of the responses of each controller in each test of the previous figures, indicating clearly the advantage of the NEPSAC controller over the other controllers.
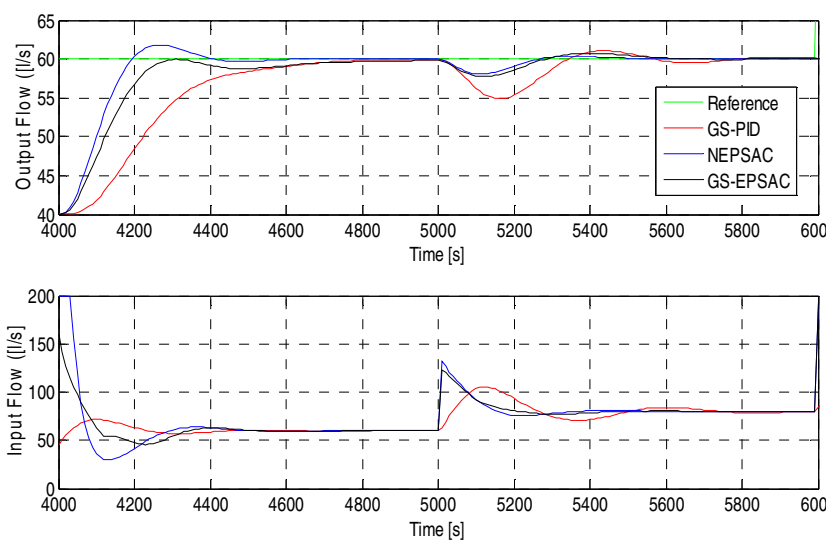

Fig. 5. Comparison of the nonlinear controllers when the reference value is 60
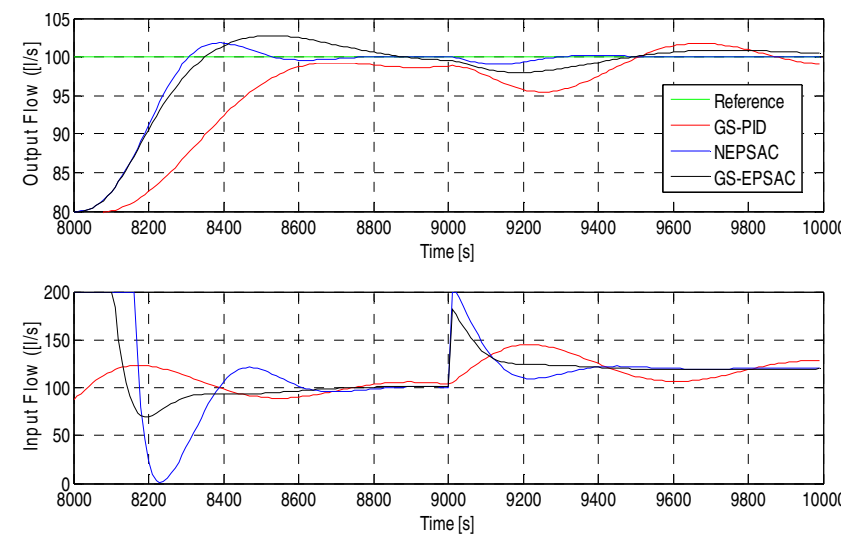

Fig. 6. Comparison of the nonlinear controllers when the reference value is 100
TABLE I. COMPARISON OF THE MSE OF THE CONTROLLERS

\begin{tabular}{|c|c|c|c|}
\hline MSE & GS-PID & NEPSAC & GS-EPSAC \\
\hline$r=20$ & 12.94 & 6.71 & 8.84 \\
\hline$r=60$ & 38.36 & 16.71 & 20.24 \\
\hline$r=100$ & 61.22 & 30.57 & 32.71 \\
\hline
\end{tabular}

\section{CONCLUSIONS}

1) Three nonlinear control techniques were applied to a three-tank system, a very nonlinear process. They present acceptable responses in the entire operation range, with the NEPSAC controller showing the best response for setpoint tracking as well as for disturbance rejection.

2) However, this good behavior comes with the cost of needing a nonlinear model, which in most of the cases could be difficult to identify, and also need more time of computation.

3) GS-EPSAC controller exhibits an intermediate performance, and it only uses linear models of the process around specific operating points, which can be obtained with the easier and more developed techniques to identify linear models.

4) The use of GS-PID approach leads to the worst performance of the three, but it is still acceptable and also, because of the autotuning method used to design each individual PID controller, it does not need an explicit process model, therefore, its implementation has the advantage of being the easiest and fastest of the three.

5) Finally, the application of any of these techniques will depend on the nonlinearity and available information of the system, the quality of the performance required, and the time, physical and economic resources to design the controller.

\section{REFERENCES}

[1] E. F. Camacho and C. Bordons, "Introduction to Model Based Predictive Control," in Model Predictive Control, 2nd ed., London: Springer-Verlag, 2007.

[2] E. F. Camacho and C. Bordons, "Nonlinear Model Predictive Control: an introductory review," in Assessment and Future Directions of Nonlinear Predictive Control, R. Findeisen, Berlin: Springer-Verlag, 2007.

[3] A. Grancharova and T. A. Johansen, "Nonlinear Model Predictive Control," in Explicit Nonlinear Model Predictive Control, Berlin: Springer-Verlag, 2012.

[4] R. De Keyser, "Model Based Prediction Control for Linear Systems," UNESCO Encyclopaedia of Life Support Systems, Article contribution 6.43.16.1, Oxford, 2003.

[5] R. De Keyser and A. Hernandez, "Evaluation of the NEPSAC nonlinear predictive controller on a thermal process," Control Conference (ECC), 2014 European, Strasbourg, 2014, pp. 814-819.

[6] W. Ipanaqué, J. Oliden, J. Manrique, A. Hernández, A. Dutta, and R. De Keyser, "Nonlinear predictive control of an evaporator for bioethanol production," in 2013 European Control Conference (ECC), Zurich, 2013, pp. 2573-2578.

[7] W. J. Rugh, J. S. Shamma, "Research on gain scheduling," Automatica, Volume 36, Issue 10, October 2000, pp. 1401-1425.

[8] R. De Keyser and A. R. Van Cauwenberghe, "Extended prediction selfadaptive control," in IFAC Symp. on Identification and System Parameter Estimation, York, 1985, pp. 1255-1260.

[9] R. De Keyser and C. M. Ionescu, "A comparative study of three relaybased PID-autotuners," IASTED Asian Conf. on Modeling, Identification and Control, Phuket, 2010, pp. 303-310 